\title{
Variations in birth weight within the normal range are related to visual orienting in infancy for boys but not for girls
}

\author{
James L. Dannemiller \\ Room 521, Waisman Center, University of Wisconsin, Madison, 1500 Highland Ave., Madison, WI 53705-2280, USA
}

Received 27 September 2002; received in revised form 24 July 2003; accepted 2 September 2003

\begin{abstract}
Pooling across 27 experiments on visual orienting during early infancy generated a large sample of full-term infants with birth weights greater than $2500 \mathrm{~g}(N=944)$. A weight-for-date measure was obtained separately for girls and for boys by residualizing birth weight on gestational age at birth. The proportion of trials with orienting toward a moving target increased directly with this weight-for-date measure for boys but not for girls in a linear regression with adjustments for gestational age at testing, birth order and visual stimulus parameters across experiments. The same relations held when raw birth weight was used as a predictor. For the 475 boys, the $z$-score of the orienting measure increased by 0.221 for each kilogram increase in birth weight corrected for gestational age $(0.196$ $z$-units per kilogram adjusted for covariates, $95 \% \mathrm{CI}=0.04-0.35 z$-units per $\mathrm{kg}, P=0.014)$. The interaction of gender and birth weight in this association makes explanations based on confounding family social variables less tenable as explanations. This association primarily for boys is similar to recently reported associations between birth weight and IQ during childhood. Possible factors underlying this association such as prenatal nutrition are discussed.
\end{abstract}

(C) 2004 Elsevier Inc. All rights reserved.

Keywords: Birth weight; Attention; Orienting; Gender

Relations between birth weight and various cognitive outcomes during childhood have been established for samples of low birth weight children $(<2500 \mathrm{~g}$; Pharoah, Stevenson, Cooke, \& Stevenson, 1994; Singer, Westphal, \& Niswander, 1968; Strauss, 2000). Do these relations between birth weight and childhood outcome measures extend into the normal birth weight range and can they also be detected during infancy? The answers appear to be yes. Visual attention measures during infancy are related to physiological maturity at term (Camp, Jamieson-Darr, Hansen, \& Schmidt, 1990). In this study, shorter length at birth was associated with longer initial fixation times on visual stimuli, and smaller head

E-mail address: dannemiller@waisman.wisc.edu (J.L. Dannemiller). 
circumference at birth was associated with smaller response decrements to repeated presentations. Longer initial fixations are more typical of younger than of older infants (Colombo, 1995), so these data suggest that individual differences in prenatal growth are related to at least one aspect of the maturity of visual attention several months later.

Visual recognition memory from 5 to 12 months of age has also been related to birth weight (Rose, 1994). In this study, the simple correlation between birth weight and visual recognition memory at 5-12 months was $r=0.26$. Additionally, after controlling for birth weight, previous illness, parental education and age-at-test, visual recognition memory was related to the infant's current weight and length. This latter relation suggests that not only weight at birth, but also that physical growth during early postnatal life is related to visual recognition memory during the latter half of the first year of life. Although no definitive statement can be made from these studies regarding the role of pre- and early postnatal nutrition, it is clear that birth weight and fetal growth are related to maternal nutrition during pregnancy (Godfrey, Robinson, Barker, Osmond, \& Cox, 1996; Harding, 2001; Lou et al., 1994).

These relations extend into childhood and beyond. Among infants born with average weights for their gestational ages, birth weight is positively related to language and gross motor development at 4 years (Ounsted, Moar, \& Scott, 1984). Birth weight in the normal range is directly related to measured intelligence in childhood and to educational attainment as late as 33 years of age (Jefferis, Power, \& Hertzman, 2002; Lagerstrom, Bremme, Eneroth, \& Janson, 1994; Matte, Bresnahan, Begg, \& Susser, 2001; Richards, Hardy, Kuh, \& Wadsworth, 2001). These relations exist even after adjustments for potentially confounding variables such as SES, maternal education, maternal age, and parity.

There is some evidence of gender differences in these outcome measures and of interactions between gender and birth weight in their relations to later outcomes. In the Camp et al. (1990) study, boys typically showed longer initial fixation times than girls; longer fixation times are usually associated with less mature attentional processing. The relation between birth weight and later IQ is stronger in males than in females (Matte et al., 2001), although the gender difference in this relation is not seen in all samples (e.g., Jefferis et al., 2002). These gender differences also raise interesting questions about the factors that produce the association between birth weight and later cognitive outcomes. Boys in general are more susceptible than girls to the deleterious effects of prenatal factors such as maternal smoking and other drug use (Moe \& Slinning, 2001; Spinillo et al., 1994; Wakschlag \& Hans, 2002). Birth weight, especially low birth weight $(<2500 \mathrm{~g})$, is related more strongly for boys than for girls to subsequent physical and psychological problems such as sudden infant death syndrome, impaired respiratory function, conduct disorder, early drug use, depression and learning disabilities (Chilcoat \& Breslau, 2002; Johnson \& Breslau, 2000; Lum et al., 2001; Saugstad, 1997; Thompson, Syddall, Rodin, Osmond, \& Barker, 2001).

The goal in this research was to examine the relations between birth weight in the normal range and attention during early infancy. Simple visual orienting behavior is one component of attention that is relatively easy to observe and to measure during infancy. This early orienting behavior probably plays a role in the development of more complex attentional systems that emerge later in infancy and mature into early childhood (Posner, Rothbart, \& Thomas-Thrapp, 1997). When prematurity is complicated by other factors such as intraventricular hemorrhage, visual orienting is affected, but more sustained attention is not (Landry, Leslie, Fletcher, \& Francis, 1985). A second goal was to search for evidence during this earlier period of the gender difference noted above in the relation between birth weight and cognitive outcomes. Positive evidence of this relation and of the gender difference in this relation would constrain the kinds of explanations that might be useful in understanding the observed association between birth weight and later attentional and cognitive development. 


\section{Method}

\subsection{Sample}

To examine the relation between birth weight and visual orienting, I pooled across 27 experiments that had been conducted in my lab between the years of 1996 and 2001 (Dannemiller, 1998, 2000, 2002; Dannemiller \& Stephens, 2001; Ross \& Dannemiller, 1999). These experiments typically had small samples (e.g., approximately 20-32 subjects), so that subtle effects of birth weight and birth order were not tested in the individual samples. Various manipulations of stimulus parameters were used in these studies, but they all involved a common outcome measure: orienting toward a moving bar amidst potentially distracting static bars (see Section 1.2). The final sample comprised 944 infants each tested once between approximately 2 and 5 months postnatally of whom 469 were girls. Infants were screened to have been born within \pm 2 weeks of their due dates as measured from the date of last menses and to have had no major birth complications nor spent any time in a neonatal intensive care unit. ${ }^{1}$ Only infants with birth weights greater than $2500 \mathrm{~g}$ were included in the analyses. Birth weight and gestational age (postnatal age corrected for the number of days early or late relative to the expected due date) were based on parental report. ${ }^{2}$ Males were also screened for a family history of color blindness, and if there was one, then these infants did not participate. Data collected on a small $(n=13)$ sample of boys with a family history of color blindness indicated that they did not differ in terms of average birth weight, average birth order or average length of gestation from boys without a family history of color blindness tested at similar ages. It is unlikely that this selection factor was responsible for the gender difference reported in the following. Table 1 shows the sample characteristics.

\subsection{Measurement of visual orienting}

Infants were shown repeated trials of a display with small, vertical bars randomly distributed across the extent of the display. Only one of these bars either on the right or the left side of the screen oscillated in place horizontally through approximately $0.5-1^{\circ}$ at a rate from 1.2 to $2.4 \mathrm{~Hz}$ across the various experiments. An observer who was unaware of the location of the moving bar made a forced choice, right versus left judgment of the location of the moving bar based on the infant's initial visual orienting, and she was given feedback after each trial regarding the correctness of the judgment. A correct judgment was one

\footnotetext{
${ }^{1}$ Using date of last menses (DLM) to estimate the due date has some error associated with it. When compared to ultrasonography, some studies show that ultrasonography is slightly more accurate than DLM (Nguyen, Larsen, Engholm, \& Moller, 1999). The average error in the predicted due date with ultrasonography was 7.96 days, and with DLM it was 8.63 days-a difference of less than 1 day between the methods. Other studies show essentially no difference in the accuracy of the methods (Olsen \& Clausen, 1998). In this latter study, DLM predicted on average 3.3 days earlier than actual delivery and ultrasonography predicted 2.0 days after actual delivery. The effect of this error in our analyses would be to weaken the potential associations between any outcome and any measure based on estimated due date, e.g., weight-for-date and gestational age at testing.

${ }^{2}$ There is bound to be error in parental reports of birth weight and gestational age. Seidman, Slater, Ever-Hadani and Gale (1987) compared maternal reports of birth weight and gestational age to actual birth records. Seventy-five percent of maternal reports were accurate to within $100 \mathrm{~g}$ on average for birth weight and to within 1 week on average for gestational age (days preor post-term relative to the expected due date). Seidman et al. (1987) asked for recall of all births for a given mother, and the authors reported that the most recent birth was recalled most accurately. This would apply to our samples because we were testing the most recently born infant for a given mother. The authors concluded that maternally reported birth weights and gestational ages are sufficiently accurate for clinical and epidemiological use.
} 
Table 1

Sample characteristics, mean (S.D.)

\begin{tabular}{lcc}
\hline & Boys & Girls \\
\hline Sample size & 475 & 469 \\
Birth weight (g) & $3688(453)$ & $3539(421)$ \\
Birth order & $1.93(1.01)$ & $1.84(0.96)$ \\
Gestational age at testing (days) & $105.7(23.1)$ & $106.4(22.6)$ \\
Gestation duration (term = 0 days) & $-0.26(6.47)$ & $-0.88(6.81)$ \\
Proportion correct orienting & $0.71(0.12)$ & $0.70(0.12)$ \\
Response time (s) & $1.85(0.38)$ & $1.82(0.37)$ \\
\hline
\end{tabular}

which corresponded to the right versus left position of the single moving target. The judgments were made quickly after the onset of the display with the average judgment taking $1.83 \mathrm{~s}$ (S.D. $=0.38 \mathrm{~s}$ skew $=1.153$ ). Infants were shown from 48 to 72 of these trials with the number of bars on the display ranging from 2 to 28 , and with the two sides of the display always having the same number of bars. The proportion of correct judgments was used as the outcome measure in the analyses given in the following. Values near 0.5 indicate that orienting was random with respect to the location of the moving target. Values near 1.0 indicate selective orienting to the oscillating target independently of the accompanying static "distractors." The split-half reliability of this orienting score was moderate, $r(941)=0.492, P<0.001$, two-tailed, and it did not differ between the genders (males $r(472)=0.489$, females $r(467)=0.496$ ). It should be noted that this moderate reliability of the outcome measure can place an upper limit on the estimated correlation between birth weight and orienting.

The orienting measure itself probably reflects at least two aspects of the infant's behavior. First, it probably reflects sensitivity to the movement itself which changes over this age range (Roessler \& Dannemiller, 1997). Second, it probably reflects consistency in orienting to the moving target. There is no premium for the infant to orient toward the side with the moving target. There are multiple potential saccadic targets in the visual field. The only thing that distinguishes the target bar from the other bars in the field is that it is the only one that is moving. The infant has a "choice" as it were on each trial to orient toward the moving bar or toward one of the other static bars. Consistency in this orienting may also reflect the extent to which the moving bar perceptually "pops-out" from its surrounding static bars.

\subsection{Statistical analysis}

The General Linear Model (GLM) was used to examine the relations between birth weight and the proportion of correct orienting trials. Study and gender were used as fixed factors. Study referred to the particular one of the 27 experiments in which the infant had participated. Because these experiments had different stimulus parameters (e.g., temporal oscillation rates, amplitudes of oscillation, contrasts of the bars) study was used as an independent factor to control for variance attributable to these stimulus factors. Covariates were also used to test relations with birth weight, birth order and gestational age at testing. Additionally, several individual difference variables computed from the infant's testing session were also used as covariates including the average response time for the observer's judgments and the degree of rightward orienting bias across trials shown by each infant. The only effect of including these additional covariates was an increase in the percentage of explained variance. The results with respect to birth weight 
Table 2

Intercorrelations separately by gender for birth weight (BWTGRAM), length of gestation (relative to expected delivery date; TERM), and the residual of birth weight regressed on length of gestation (residual for BWTGRAM)

\begin{tabular}{rlc}
\hline Females $(n=469)$ & & Residual for BWTGRAM \\
Residual for BWTGRAM & 1.000 & $.970^{* *}$ \\
BWTGRAM & $.970^{* *}$ & 1.000 \\
TERM & .000 & $.244^{* *}$ \\
Males $(n=475)$ & & BWTGRAM \\
Residual for BWTGRAM & Residual for BWTGRAM & $.958^{* *}$ \\
BWTGRAM & 1.000 & 1.000 \\
TERM & $.958^{* *}$ & $.285^{* *}$ \\
\hline
\end{tabular}

** Correlation is significant at the 0.01 level (two-tailed).

and gender did not change meaningfully with these latter two individual difference covariates included in the analysis, so the results reported in the following only include birth weight, birth order and gestational age at testing as covariates and gender and study as fixed factors. The interactions of gender with birth weight, birth order and gestational age at testing were also tested. Separate analyses were conducted on girls and boys after it had been established that there was a significant interaction between birth weight and gender in the initial GLM.

Birth weight was recorded in pounds and ounces from parental report and converted to grams. A more direct measure of fetal growth can be obtained by residualizing birth weight on length of gestation. This adjusts for the small, but significant positive relation between length of gestation and birth weight. The results reported below used this weight-for-date (WFD) measure, but the analyses were also conducted using unadjusted birth weight, and they were substantively the same. It can be seen from Table 2 that even after residualizing birth weight on length of gestation, there was still a near perfect correlation between birth weight and this residualized measure. The residualized weight-for-date measure was calculated separately for males and for females given the difference in expected birth weights for the two genders as a function of gestational age. The interpretations of the weight-for-date and the birth weight measures are slightly different. The weight-for-date measure is positive for infants who were heavier than would have been predicted from their estimated gestational ages, and negative for infants who were lighter than would have been predicted from their estimated gestational ages. The raw birth weight measure is interpreted more straightforwardly and independently of gestational age. Given the high correlation between these two measures as shown in Table 2, it matters very little whether the results are interpreted in terms of raw birth weight or the residual of birth weight adjusted for length of gestation.

\section{Results}

Significant effects were found for study, $F(26,911)=28.72, P<0.001$, gestational age at testing, $F(1,911)=151.22, P<0.001$, and the interaction of gender and WFD, $F(1,911)=6.73, P=0.010$. None of the other main effects (gender, birth order, WFD) nor the interactions (gender $\times$ birth order and gender $\times$ gestational age at testing) were significant (all $P$ 's $>0.29$ ). Thus, the overall proportion of trials with correct orienting varied significantly with the particular stimulus parameters that were used (study), 
with the gestational age at which the infant was tested, and with WFD, but the last effect depended on the infant's gender. For every additional week of gestational age at the time of test, the $z$-score of the orienting measure increased on average by $0.12(95 \% \mathrm{CI}=0.097-0.134, P<0.001)$.

Given the significant interaction of gender and WFD, separate analyses were conducted for boys and girls using the same covariates as in the previous analyses with gender removed as a fixed factor. For girls, the simple correlation between WFD and the proportion of trials with correct orienting was not significantly different from $0, r(467)=-0.05, P=0.278$, two-tailed. This relation remained nonsignificant when variance attributable to study, birth order and gestational age at testing was controlled.

For boys, the simple correlation between WFD and the proportion of trials with correct orienting was significantly different from $0, r(473)=0.098, P=0.033$, two-tailed. This relation increased in significance when variance attributable to study, birth order and gestational age at testing was controlled. The change in the $z$-score of the orienting measure for each kilogram increase in birth weight corrected for gestational age for boys was significantly greater than $0.0(0.196,95 \% \mathrm{CI}=0.04-0.35, P=0.014)$. By way of comparison, when the birth weight was left uncorrected for length of gestation, the simple correlation between birth weight and orienting score for boys was $r(473)=0.118, P=0.010$. For girls this uncorrected correlation was $r(467)=-0.034, P=0.461$. For boys the association was somewhat stronger when birth weight was left uncorrected for length of gestation implying that some of the variance in the orienting score explained by birth weight was associated with length of gestation as well.

The effect size in the sample of boys was small, and the sample itself was large. To what extent is this effect still significant with smaller samples? A split-half analysis was conducted to determine the robustness of the effect. Two half-samples were created by randomly splitting the samples in each of the contributing experiments, and separate analyses identical to those reported above were conducted on these two half-samples. Only infants with birth weights less than $4500 \mathrm{~g}$ were included because of the possible nonlinear effect above $4500 \mathrm{~g}$ evident in Fig. 1. In both half-samples, the weight-for-date measure was a significant positive predictor of the attentional measure for boys $(P=0.02$ and 0.05$)$. In neither half sample, was this weight-for-date measure a significant predictor of the attentional measure in girls $(P=0.663$ and 0.285$)$.

It was also the case that the mean percentage of correct orienting responses was nearly identical between the two genders (males, $M=0.71$; females, $M=0.70$ ). This implies that the linear trend with birth weight for boys must start with the lighter boys having lower orienting scores than the girls with similar birth weights, and the heavier boys showing higher orienting scores than girls with similar birth weights. This was indeed the case. Fig. 1 shows birth weight divided into five contiguous categories with the mean orienting score for each category plotted separately for girls and for boys. Although the differences are slight, the trend is evident across the range of birth weights for boys, but not for girls. There is some indication for both genders that there is a decline in orienting at the heaviest birth weights $(>4500 \mathrm{~g})$, although the sample sizes are small in this range, so this trend is only suggested by an inspection of the data.

It is important to establish that the lack of an association in females was not a result of reduced variance in birth weight or on the orienting measure. As shown in Table 1, the standard deviations of birth weights were similar for the two sexes (males, S.D. $=453 \mathrm{~g}$; females, S.D. $=421 \mathrm{~g}$ ). The ranges were also similar (males, range $=2608 \mathrm{~g}$, females, range $=2495 \mathrm{~g}$ ). On the orienting measure, the standard deviations $($ S.D. $=0.12$ ) and the ranges (range $=0.55$ ) were identical for boys and girls. Additionally, when the analysis was restricted to males within the range of females' birth weights, the same relation with birth weight (weight-for-date) was observed, $F(1,443)=6.37, P=0.012$. Thus, it is unlikely that 


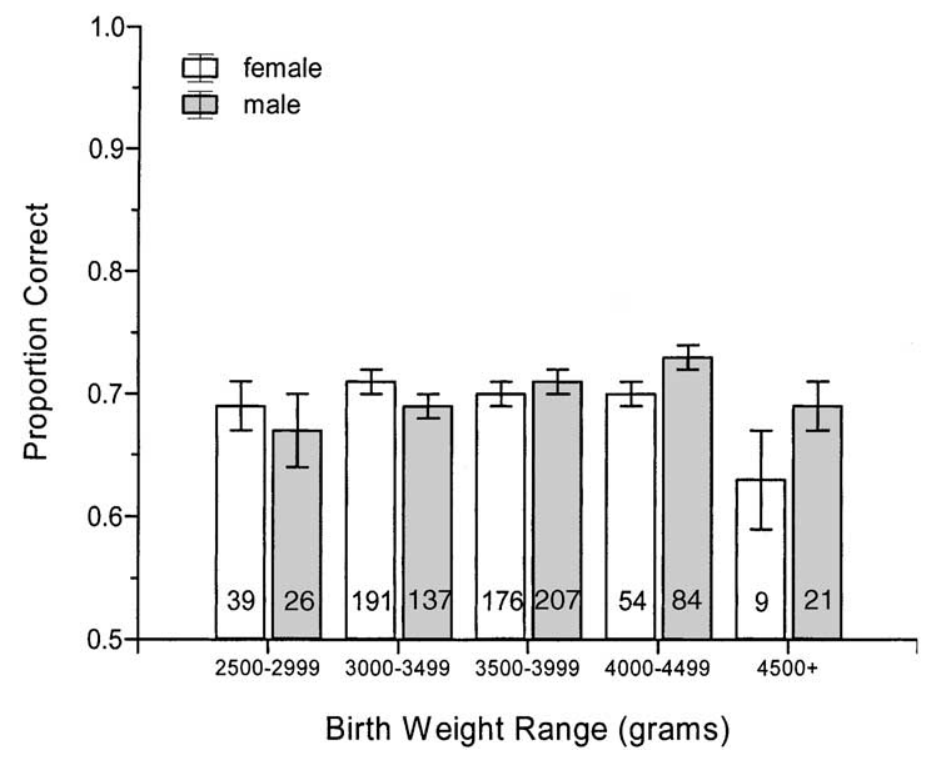

Fig. 1. Mean proportion correct orienting for boys and girls plotted by birth weight category. The error bars are \pm S.E.M. The number within each bar is the sample size in that category.

differences in the variances of the birth weight and orienting variables are responsible for the larger effect in males than in females, a conclusion reinforced by the results shown in Fig. 1 indicating that the trend exists across the birth weight range for boys.

Finally, because the distributions of birth weights differ systematically by gender, it is possible that using an absolute scale to define the normal birth weight range could lead to more growth-retarded boys than girls and more large-for-date girls than boys in the sample. The trend of the attentional measure across birth weight in males could potentially be attributable to the excess of growth-retarded boys at the lower end of the male birth weight distribution. To address this potential alternative explanation, two restricted subsamples were created. First, the bottom 90th percentile by birth weight of the female sample was used to eliminate a potential relative excess of large-for-date females. The $90 \%$ for females by birth weight was $3005 \mathrm{~g}$, and this resulted in a subsample with $N=418$ females. Next, the bottom $10 \%$ by birth weight of the male distribution plus male infants weighing more than $4500 \mathrm{~g}$ were eliminated from the male sample. The 10th percentile of male birth weight was $3090 \mathrm{~g}$. This left a subsample of $N=404$ males with birth weights in the normal range eliminating a potential excess of growth-retarded males as well as the large-for-gestational age $(>4500 \mathrm{~g})$ males.

Identical analyses were conducted on these two subsamples using the same fixed factor (study) and the same covariates (birth order, gestational age at testing, and residual weight-for-date adjusted separately for males and females as described above). The weight-for-date measure remained a significant predictor of the attentional measure in the male sample, $F(1,374)=4.55, P=0.034$. The weight-for-date measure was not a significant predictor of the attentional measure in the female subsample, $F(1,388)=0.243$, $P=0.622$. Additionally, the simple correlation between birth weight and the attentional measure was significant for the male subsample, $r(402)=0.128, P=0.01$, two-tailed, but it was not significant in the female subsample, $r(416)=-0.004, P=0.929$. These analyses show that the same relation between 
birth weight and the attentional measure again holds only for males when the differential distribution of birth weights by gender is taken into account.

\section{Discussion}

Visual orienting from 2 to 5 months of age is related to birth weight for boys, but not for girls. This relation for boys only held from 2500 to $4500 \mathrm{~g}$. Above a birth weight of $4500 \mathrm{~g}$, there was a trend for both genders toward lower scores on this orienting measure than the scores exhibited by their adjacent cohorts weighing just under $4500 \mathrm{~g}(4000-4499 \mathrm{~g})$. The positive relation between birth weight and orienting in infancy for boys is similar to previous reports showing a stronger association between birth weight and IQ in childhood for boys than for girls (Matte et al., 2001). The effect size in the current study, an increase of approximately one-fifth of a $z$-unit per kilogram of additional birth weight corrected for gestational age separately by gender, is remarkably similar to the effect sizes reported in the studies on childhood IQ using large samples (Jefferis et al., 2002; Matte et al., 2001; Richards et al., 2001). These effect sizes ranged from 0.14 to $0.33 z$-units per kilogram increase in birth weight.

It is worth noting that the effect size for this association is meaningful mostly for what it might tell us about the factors operating in the population. An effect size of one-fifth of a $z$-unit per kilogram of birth weight is too small to be meaningful at the level of the individual child. If the orienting measure were analogous to an IQ test with a standard deviation of 15 points, then this effect size would amount to three IQ points per kilogram of additional birth weight. The value in knowing this association is that it may hold clues for our understanding of how various prenatal factors distributed in the population are related to behavioral outcomes in infancy and childhood.

There are several questions that arise when considering these results. First, why should variations in birth weight be related to visual orienting? Second, why should this relation hold more strongly for boys than for girls? The first question leads to a consideration of the factors that influence birth weight. The second question leads to a consideration of factors that might operate differentially by gender during prenatal development.

How could birth weight be related to cognitive performance whether during infancy or childhood? Both variables involved in this association could be related to a third variable such as SES. As noted above, the relation between birth weight and childhood IQ persists in identical twins and same-sex siblings despite essentially equal SES environments (Churchill, 1965; Matte et al., 2001; Scarr, 1969; Willerman \& Churchill, 1967). It is worth noting that the observation of this association in pairs of identical twins also rules out a genetic basis for the relation. The association must be produced by some other prenatal process that is more directly related to birth weight. One such candidate process is prenatal brain development. Birth weight and head circumference are correlated (Crawford, Doyle, \& Meadows, 1987; Nishi et al., 1992), and head circumference at birth reflects the rate of head growth in utero (Sheth, Mullett, Bodensteiner, \& Hobbs, 1995). Childhood IQ and attention in infancy are not just related to birth weight, but also to head circumference at birth (Camp et al., 1990; Ounsted et al., 1984). To the extent that head circumference is an indirect measure of brain size, then this association between birth weight and later cognition may reflect the physiological maturity of the brain at birth.

Prenatal nutrition plays a key role in fetal growth. The timing of nutrient intake also appears to be related to fetal growth. High maternal carbohydrate intake early in pregnancy or low dairy and meat protein intake late in pregnancy both tend to result in lower birth weight (Godfrey et al., 1996). Maternal weight gain 
during pregnancy and fasting glucose production during the third trimester of pregnancy are also related to estimated fetal weight in the third trimester (Rohl, Huston-Presley, Amini, Stepanchak, \& Catalano, 2001). There is growing evidence that the timing of malnutrition or under-nutrition during gestation can predispose the offspring to diseases during adulthood - a concept known as fetal programming (Harding, 2001). For example, rates of death from coronary heart disease are inversely related to birth weight through $4224 \mathrm{~g}$, and interestingly, this association appeared to be stronger for men than for women (Dennison, Fall, Cooper, \& Barker, 1997). The fetus may be able to compensate at least partially for maternal undernutrition by switching to alternative fuel sources for oxidative metabolism, but there can be no doubt that the compensation is not complete (Adam, Raihaa, Rahiala, \& Kekomaki, 1975). It is reasonable to suppose that a similar nutritional mechanism might influence the prenatal development of the brain.

The puzzling aspect of these results and prior results is the role of gender in this relation. If brain maturity at birth underlies the relation between birth weight and visual orienting during infancy, then why should this relation depend on gender? One answer is that differences in the rate of growth between males and females prenatally might leave male growth more susceptible to variations in nutrition or to subtle insults during gestation.

Boys grow faster on average than girls during prenatal development (Crawford et al., 1987). The evidence for this is that on average, boys tend to be heavier than girls at birth. In the current sample, the average difference in birth weight was $149 \mathrm{~g}$ in favor of the boys. This is slightly larger than the difference reported in one published study - approximately $90 \mathrm{~g}$ - for infants born within the range of normal birth weights (e.g., Matte et al., 2001), and slightly smaller than the difference reported in another study - approximately $183 \mathrm{~g}$ (Godfrey et al., 1996). Since average gestational age at birth does not differ between the genders, by definition, boys must have been growing faster on average during gestation. An alternative measure of growth rate that supports the same conclusion is the gestational age at which infants are born with a given weight; boys on average are gestationally younger than girls for a given birth weight (de Zegher, Devlieger, \& Eeckels, 1999). In other words, on average boys reach a given body weight earlier in gestation than do girls. This is especially true at lower birth weights which may partly explain why premature birth is more of a risk factor for later physical and psychological problems for boys.

How is this greater average birth weight of boys accomplished? Tamimi et al. (2003) showed that mothers pregnant with boys have approximately a $10 \%$ greater energy intake than those pregnant with girls. Thus, it is primarily maternal nutrition during pregnancy that is responsible for the faster growth rates and heavier birth weights of boys than of girls.

What role could the faster rate of prenatal growth in boys have in explaining the gender difference in the association between birth weight and childhood IQ and between birth weight and orienting during infancy? Other domains may hold clues to the nature of this association. Sex differences in coronary artery disease during adult life have been related to differences in birth measurements (Forsen, Ericksson, Osmond, \& Barker, 1999). The authors of that study suggested that differences in fetal growth rates could lead to different responses to fetal undernutrition. The hypothesis is that boys are growing more rapidly at least at some points in prenatal development, and as such, they may be more susceptible to the influences of nutritional variation during gestation. Two of the principles of fetal programming are that (a) rapidly growing fetuses are more sensitive to the effects of undernutrition, and (b) the permanent effects of undernutrition include reduced cell number and altered organ structure (Dennison et al., 1997). This may explain why variations in birth weight among boys are more strongly related to later cognitive outcomes than among girls. 
In summary, visual orienting measured during the period from 2 to 5 months postnatally in a sample of full term infants born with normal birth weights is associated positively with birth weight adjusted for length of gestation for boys but not for girls. This gender difference is similar to one shown in another report in which birth weight was more strongly related to IQ in childhood for boys than it was for girls (Matte et al., 2001). The role of gender in this association makes explanations based on SES or other confounding family variables less tenable. As late as 5 months of age, visual orienting in boys reflects in a small but significant way their weights at birth. Girls orient on average as well as boys, but their birth weights are unrelated to their visual orienting over this age range. The factors underlying this interaction between gender and birth weight remain to be determined. The current study can only speculate on the potential role of nutrition in these relations because data on prenatal nutrition were not collected. The lower scores of the macrosomic infants of both genders (birth weights greater than $4500 \mathrm{~g}$ ) in the current study are intriguing in this regard. Current research is under way in our lab to gather more precise details on the pregnancies of these mothers of macrosomic infants.

\section{Acknowledgements}

This research was supported by NICHD R01 HD32927 and a University of Wisconsin Vilas Foundation Fellowship to JLD. I thank Jacqueline Roessler and Shannon Ross for observing the infants, Manya Qadir for scheduling the infants, Daniel Replogle for all of the computer programming, Rebecca Seltzer and Kate Hobbs for data entry, and Emily Dimond for helpful discussions on macrosomia and diabetes. Kate Hobbs was supported by a Praxis Fellowship from Smith College.

\section{References}

Adam, P. A. J., Raihaa, N., Rahiala, E.-L., \& Kekomaki, M. (1975). Oxidation of glucose and D-B-OH-butyrate by the early human fetal brain. Acta Paediatrica Scandinavica, 64, 17-24.

Camp, B. W., Jamieson-Darr, K., Hansen, R., \& Schmidt, B. (1990). Growth parameters and attention to faces at 4 to 6 months of age. Journal of Developmental and Behavioral Pediatrics, 11, 229-233.

Chilcoat, H. D., \& Breslau, N. (2002). Low birth weight as a vulnerability marker for early drug use. Experimental and Clinical Psychopharmacology, 10, 104-112.

Churchill, J. A. (1965). The relationship between intelligence and birth weight in twins. Neurology, 15, $341-347$.

Colombo, J. (1995). On the neural mechanisms underlying developmental and individual differences in infant fixation duration: Two hypotheses. Developmental Review, 15, 97-135.

Crawford, M. A., Doyle, W., \& Meadows, N. (1987). Gender differences at birth and differences in fetal growth. Human Reproduction, 2, 517-520.

Dannemiller, J. L. (1998). A competition model of exogenous orienting in 3.5-month-old infants. Journal of Experimental Child Psychology, 68, 169-201.

Dannemiller, J. L. (2000). Competition in early exogenous orienting between 7 and 21 weeks. Journal of Experimental Child Psychology, 76, 253-274.

Dannemiller, J. L. (2002). Relative color contrast drives competition in early exogenous orienting. Infancy, 3, $275-301$.

Dannemiller, J. L., \& Stephens, B. R. (2001). Asymmetries in contrast polarity processing in young human infants. Journal of Vision, 1(2), 112-125 [On-line]. Available at: http://journalofvision.org/1/2/5 (DOI 10.1167/1.2.5).

Dennison, E., Fall, C., Cooper, C., \& Barker, D. (1997). Prenatal factors influencing long-term outcome. Hormone Research, $48,25-29$.

de Zegher, F., Devlieger, H., \& Eeckels, R. (1999). Fetal growth: Boys before girls. Hormone Research, 51, $258-259$. 
Forsen, T., Ericksson, J. G., Osmond, C., \& Barker, D. J. P. (1999). Growth in utero and during childhood among women who develop coronary heart disease: Longitudinal study. British Medical Journal, 319, 1403-1407.

Godfrey, K., Robinson, S., Barker, D. J. P., Osmond, C., \& Cox, V. (1996). Maternal nutrition in early and late pregnancy in relation to placental and fetal growth. British Medical Journal, 312, 410-414.

Harding, J. E. (2001). The nutritional basis of the fetal origins of adult disease. International Journal of Epidemiology, 30 , 15-23.

Jefferis, B., Power, C., \& Hertzman, C. (2002). Birth weight, childhood socioeconmic environment, and cognitive development in the 1985 British birth cohort study. British Medical Journal, 325, 305-310.

Johnson, E. O., \& Breslau, N. (2000). Increased risk of learning disabilities in low birth weight boys at age 11 years. Biological Psychology, 47, 490-500.

Lagerstrom, M., Bremme, K., Eneroth, P., \& Janson, C.-G. (1994). Long-term development for girls and boys at age 16-18 as related to birth weight and gestational age. International Journal of Psychophysiology, 17, 175-180.

Landry, S. H., Leslie, N. A., Fletcher, J. M., \& Francis, D. J. (1985). Visual attention skills of premature infants with and without intraventricular hemorrhage. Infant Behavior and Development, 8, 309-321.

Lou, H. C., Hansen, D., Nordentoft, M., Pryds, O., Jensen, F., Nim, J., \& Hemmingsen, R. (1994). Prenatal stressors of human life affect fetal brain development. Developmental Medicine and Child Neurology, 36, 826-832.

Lum, S., Hoo, A.-F., Dezateux, C., Goetz, I., Wade, A., DeRooy, L., Costeloe, K., \& Stocks, J. (2001). The association between birthweight, sex, and airway function in infants of nonsmoking mothers. American Journal of Respiratory and Critical Care Medicine, 164, 2078-2084.

Matte, T., Bresnahan, M., Begg, M., \& Susser, E. (2001). Influence of variation in birth weight within normal range and within sibships on IQ at age 7 years: Cohort study. British Medical Journal, 323, 310-314.

Moe, V., \& Slinning, K. (2001). Children prenatally exposed to substances: Gender-related differences in outcome from infancy to 3 years of age. Infant Mental Health Journal, 22, 334-350.

Nguyen, T. H., Larsen, T., Engholm, G., \& Moller, H. (1999). Evaluation of ultrasound-estimated date of delivery, ultrasound in obstetrics in 17,450 spontaneous singleton births: Do we need to modify Naegele's rule? Ultrasound in Obstetrics and Gynecology, 14, 23-28.

Nishi, M., Hirotsugu, M., Hirofumi, A., Shimizu, H., Tatetama, H., Chaki, R., Tsukuda, H., Nomura, H., Hatanaka, Y., \& Nishi, M. (1992). An index for proportion of head size to body mass during infancy. Journal of Child Neurology, 7, 400-403.

Olsen, O., \& Clausen, J. A. (1998). Determination of the expected day of delivery-Ultrasound has not been shown to be more accurate than the calendar method. Ugeskrift for Laeger, 160, 2088-2090.

Ounsted, M., Moar, V. A., \& Scott, A. (1984). Associations between size and development at four years among children who were small-for-dates and large-for-dates at birth. Early Human Development, 9, 259-268.

Pharoah, P. O., Stevenson, C. J., Cooke, R. W., \& Stevenson, R. C. (1994). Clinical and subclinical deficits at 8 years in a georgraphically defined cohort of low birthweight infants. Archives of Disease in Childhood, 70, 264-270.

Posner, M. I., Rothbart, M. K., \& Thomas-Thrapp, L. (1997). Functions of orienting in early infancy. In: P. J. Lang, R. F. Simons, \& M. Balaban (Eds.), Attention and orienting (pp. 327-345). Mahwah, NJ: Lawrence Erlbaum Associates.

Richards, M., Hardy, R., Kuh, D., \& Wadsworth, M. (2001). Birth weight and cognitive function in the British 1946 birth cohort: Longitudinal population based study. British Medical Journal, 322, 199-203.

Roessler, J., \& Dannemiller, J. (1997). Changes in human infants' sensitivity to slow displacements over the first 6 months. Vision Research, 37, 417-423.

Rohl, J., Huston-Presley, L., Amini, S., Stepanchak, B., \& Catalano, P. (2001). Factors associated with fetal growth and body composition as measured by ultrasound. American Journal of Obstetrics and Gynecology, 185, 1416-1420.

Rose, S. A. (1994). Relation between physical growth and information processing in infants born in India. Child Development, 65, 889-902.

Ross, S. M., \& Dannemiller, J. L. (1999). Color contrast, luminance contrast and competition within exogenous orienting in 3.5-month-old infants. Infant Behavior and Development, 22, 383-404.

Saugstad, L. F. (1997). Optimal foetal growth in the reduction of learning and behaviour disorder and prevention of sudden infant death (SIDS) after the first month. International Journal of Psychophysiology, 27, 107-121.

Scarr, S. (1969). Effects of birth weight on later intelligence. Social Biology, 16, 249-256.

Sheth, R. D., Mullett, M. D., Bodensteiner, J. B., \& Hobbs, G. R. (1995). Longitudinal head growth in developmentally normal preterm infants. Archives of Pediatrics and Adolescent Medicine, 149, 1358-1361.

Seidman, D. S., Slater, P. E., Ever-Hadani, P., \& Gale, R. (1987). Accuracy of mothers' recall of birthweight and gestational age. British Journal of Obstetrics and Gynaecology, 94, 731-735. 
Singer, J. E., Westphal, M., \& Niswander, K. R. (1968). Sex differences in the incidence of neonatal abnormalities and abnormal performance in early childhood. Child Development, 38, 103-112.

Spinillo, A., Capuzzo, E., Nicola, S., Colonna, L., Iasci, A., \& Zara, C. (1994). Interaction between fetal gender and risk factors for fetal growth retardation. American Journal of Obstetrics and Gynecology, 171, 1273-1277.

Strauss, R. S. (2000). Adult functional outcome of those born small for gestational age: Twenty-six-year follow-up of the 1970 British birth cohort. Journal of the American Medical Association, 283, 625-632.

Tamimi, R. M., Lagiou, P., Mucci, L. A., Hsieh, C. C., Adami, H. O., \& Trichopoulos, D. (2003). Average energy intake among pregnant women carrying a boy compared with a girl. British Medical Journal, 326, 1245-1246.

Thompson, C., Syddall, H., Rodin, I., Osmond, C., \& Barker, D. (2001). Birth weight and the risk of depressive disorder in late life. British Journal of Psychiatry, 179, 450-455.

Wakschlag, L. S., \& Hans, S. L. (2002). Maternal smoking during pregnancy and conduct problems in high-risk youth: A developmental framework. Development and Psychopathology, 14, 351-369.

Willerman, L., \& Churchill, J. A. (1967). Intelligence and birth weight in identical twins. Child Development, 38, 623-629. 